\title{
Efeitos da hipertensão e do treinamento aeróbio sobre a expressão de diferentes componentes da barreira hematoencefálica.
}

Mestrando: Matheus Garcia de fragas

Orientadora: Lisete Compagno Michelini

\section{RESUMO}

Fragas MG. Efeitos da hipertensão e do treinamento aeróbio sobre a expressão de diferentes componentes da barreira hematoencefálica. [Dissertação (Mestrado em Ciências - Fisiologia humana) ]. São Paulo. Instituto de Ciências Biomédicas. Universidade de São Paulo; 2018.

A hipertensão arterial cursa com disfunção autonômica e lesão da barreira hematoencefálica (BHE) em áreas de controle autonômico. Demonstramos recentemente que o treinamento aeróbio corrige a lesão da BHE, e a disfunção autonômica, a qual se encontra correlacionada com a integridade da BHE observada nos hipertensos treinados. O objetivo deste trabalho é avaliar a expressão gênica e proteica de componentes da BHE envolvidos na mediação das respostas cardiovasculares à hipertensão e ao treinamento aeróbio $(\mathrm{T})$. Ratos espontaneamente hipertensos (SHR) e seus controles normotensos (WKY) (250-300g) foram submetidos ao protocolo de T em esteira ou mantido sedentários (S) por 4 semanas. Ao final do $\mathrm{T}$ os animais dos grupos experimentais foram canulados para aquisição das variáveis hemodinâmicas. A seguir procedeu-se à infusão intra-arterial de 2 corantes (Rodamina-d, 70 KD, e FITC-d,10 KD) e 20 min após os encéfalos foram coletados para realização de ensaios de fluorescência no Núcleo Paraventricular do Hipotálamo (PVN). Outros ratos dos grupos experimentais foram perfundidos com salina via transcardíaca e realizada a microdissecção do PVN. O mRNA foi extraído e sua concentração de foi analisada pela técnica de RT-PCR. Para investigar os efeitos da hipertensão e do $\mathrm{T}$ nos componentes da BHE, foram utilizados os seguintes primers: Occludina, Claudina-5, Zônula Ocludens 1 (proteínas da junção oclusiva), Caveolina-1 (indicador de transporte transcelular), Laminina alfa 1 e Colágeno 4 (componentes da membrana basal), PDGFR $\beta$ (marcador de pericitos)e Aquaporina-4 (indicador de podócitos de astrócitos), todos eles normalizados para o HPRT endógeno. Os dados de PCR em tempo real foram quantificados pelo método $2 \Delta \Delta \mathrm{CT}$. 
Além disso, outros ratos dos mesmos grupos experimentais foram perfundidos com paraformaldeído $4 \%$ para a fixação do encéfalo. O tecido foi crioprotegido e seccionado em criostato, 30 um, os cortes foram incubados em anticorpos primários (Reca-1(marcador endotelial), Claudina-5, Caveolina-1, PDGFR $\beta$ e Aquaporina-4) e secundários (Alexa Flúor 488 e 594), e sua quantificação no PVN foi realizada através da densidade integrada. Não foi observada diferença na pressão arterial entre os grupos T e S, porém, houve bradicardia de repouso nos animais T (SHR-T:317 \pm 3 e

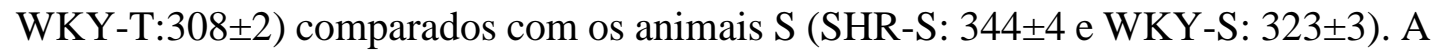
permeabilidade da BHE foi reduzida e normalizada pelo $\mathrm{T}$ nos animais hipertensos

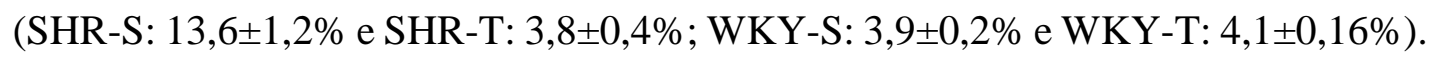
Não houve diferenças na expressão gênica de proteínas das junções oclusivas e da membrana basal, PDGFR $\beta$ e Aquaporina-4 entre os T e S, mas a expressão proteica de claudina-5 e de PDGFR $\beta$ encontravam-se aumentadas nos SHR-T vs. SHR-S. A expressão gênica e proteica de Caveolina-1 aumentada nos SHR-S vs. WKY-S, foi normalizada pelo T. Conclusão: Nossos dados sugerem que o aumento da permeabilidade da BHE no PVN de hipertensos é devido ao aumento de transcitose, identificada pela elevada expressão de Caveolina-1, sem alterações no transporte paracelular. Por sua vez e que o treinamento aeróbio reverte esta permeabilidade ao reduzir o transporte transcelular além de intensificar a função de barreira pela elevada expressão de claudina-5 e dos podócitos dos astrócitos.

Palavras chave: ratos espontaneamente hipertensos, barreira hematoencefálica, transcitose, treinamento aeróbio, hipotálamo.

\section{ABSTRACT}

Fragas MG. Effects of hypertension and aerobic training on the expression / activity of different components of the blood-brain barrier. [Masters Thesis (Master of Science - Human Physiology)]. Sao Paulo. Institute of Biomedical Sciences. University of Sao Paulo; 2018.

The arterial hypertension courses with autonomic dysfunction and Blood Brain Barrier (BBB) damage in areas of autonomic control. We recently demonstrated that aerobic training corrects the damage to the $\mathrm{BBB}$, and autonomic dysfunction, which 
is correlated with the integrity of the BBB observed in hypertensive trained. The objective of this work is to evaluate the gene and protein expression of BBB components involved in mediating cardiovascular responses to hypertension and aerobic training (T). Spontaneously hypertensive rats (SHR) and their normotensive controls (WKY) (250-300g) were submitted to treadmill protocol or maintained sedentary (S) for 4 weeks. At the end of the $\mathrm{T}$, the animals of the experimental groups were cannulated to acquire the hemodynamic variables. Intra-arterial infusion of 2 dyes (Rhodamine-d, $70 \mathrm{KD}$, and FITC-d, $10 \mathrm{KD}$ ) and $20 \mathrm{~min}$ after the brain were collected for fluorescence assays in the Paraventricular Nucleus of hypothalamus (PVN). Other rats from the experimental groups were perfused with transcardiac saline and the microdissection of PVN was performed. The mRNA was extracted and its concentration was analyzed by the RT-PCR technique. To investigate the effects of hypertension and $\mathrm{T}$ in the BBB components, the following primers were used: Occludin, Claudine-5, Zonula Ocludens 1 (tight junction proteins), Caveolina-1 (indicator of transcellular transport), Laminin alfa 1 and Collagen 4 (basement membrane components), PDGFR $\beta$ (pericyte marker) and Aquaporin-4 (astrocyte podocyte indicator), all standardized for endogenous HPRT. Real-time PCR data were quantified by the method $2 \Delta \Delta \mathrm{CT}$. In addition, other rats from the same experimental groups were perfused with $4 \%$ paraformaldehyde for fixation of the brain. The tissue was cryoprotected and cross-sectioned, $30 \mu \mathrm{m}$, sections were incubated on primary (Reca-1 (endothelial marker), Claudin-5, Caveolin-1, PDGFR $\beta$ and Aquaporin-4) and secondary antibodies (Alexa Fluor 488 and 594), and its quantification in PVN was performed through the integrated density. There was no difference in blood pressure between the $\mathrm{T}$ and $\mathrm{S}$ groups, but there was resting bradycardia in the $\mathrm{T}$ animals (SHRT: $317 \pm 3$ and WKY-T: $308 \pm 2$ ) compared to S animals (SHR-S: $344 \pm 4$ and WKY$\mathrm{S}: 323 \pm 3$ ). The permeability of $\mathrm{BBB}$ was reduced and normalized by $\mathrm{T}$ in hypertensive animals (SHR-S: $13.6 \pm 1.2 \%$ and SHR-T: $3.8 \pm 0.4 \%$; WKY-S: $3.9 \pm$ 0, $2 \%$ and WKY-T: $4.1 \pm 0.16 \%$ ). No differences were observed in gene expression of tight junctions and basement membrane proteins, PDGFR $\beta$ and Aquaporin-4 between $\mathrm{T}$ and $\mathrm{S}$, but protein expression of claudin-5 and PDGFR $\beta$ were augmented in SHR-T vs. SHR-S. Gene and protein expression of Caveolin-1, increased in the SHR-S vs. WKY-S, were normalized by T. Conclusion: Our data suggest that increased BBB permeability within the PVN of hypertensive individuals is due to increased transcytosis identified by increased expression of Caveolin-1, without 
changes in paracellular transport. In contrast, aerobic training reverses and normalizes $\mathrm{BBB}$ permeability by reducing transcellular transport, in addition to intensify barrier function by the increased expression of claudin-5 and astrocytes' endfeet.

Key-words: spontaneously hypertensive rats, blood-brain barrier aerobic training, transcytosis, hypothalamus.

\section{Introdução}

A BHE é uma interface regulatória que separa a circulação periférica do SNC, é de fundamental importância para a homeostase do SNC. É formada por um sistema celular complexo composto por células endoteliais, membrana basal, pericitos e pelos podócitos dos astrócitos que recobrem a vasculatura (2-5). Diferente dos capilares periféricos, os capilares encefálicos com BHE functional se caracterizam por não possuir fenestras, baixa atividade pinocítica e células endoteliais com a presença de junções oclusivas de alta resistência entre as células. As células endoteliais e as junções oclusivas entre as células endoteliais formam uma barreira física que bloqueia o transporte paracelular, permitindo apenas o transporte transcelular entre o capilar e o interstício através de transportadores específicos (3). As junções oclusivas são formadas por três proteínas integrais transmembrana - a Claudina-5, a Ocludina e as moléculas de adesão juncional, bem como proteínas citoplasmáticas acessórias (Zonula ocludens), estas ligam as proteínas de membrana à actina (proteína primária do citoesqueleto) mantendo a estrutura e integridade funcional do endotélio $(6,7)$.

Pequenas moléculas lipossolúveis como o óxido nítrico e os gases sanguíneos como oxigênio e dióxido de carbono idealmente difundem-se facilmente pela BHE. Por outro lado, nutrientes essenciais ao metabolismo celular como a glicose, aminoácidos e íons são captados por transportadores específicos inseridos na membrana celular, as quais são responsáveis pelo transporte de nutrientes (GLUT1, NaK-ATPase, NaKClcotransportador, receptor de insulina entre outros), como também pela remoção de produtos do metabolismo celular (Glicoproteína-P) $(3,8-11)$ 
As funções da BHE encontram-se bastante prejudicadas na hipertensão arterial (12). Sabe-se que a desregulação do fluxo sanguíneo cerebral e trocas anormais através da barreira, decorrentes da hipertensão, podem resultar na sua lesão e/ou rompimento $(9,13)$. A abertura da BHE, por sua vez, é crítica no desenvolvimento e progressão de várias doenças que afetam o SNC como o acidente vascular cerebral, a doença de Alzheimer e de Parkinson, Diabetes Mellitus do tipo 2, Esclerose Múltipla entre outras $(6,8,14-16)$

Os mecanismos através dos quais a hipertensão afeta o funcionamento da BHE ainda não são completamente conhecidos e muitas questões permanecem em aberto $(13,17,18)$. Sabe-se, porém, que a disfunção endotelial induzida por modelos de hipertensão crônica pode destruir as junções oclusivas, sendo responsável pelo aumento da permeabilidade na BHE e seu consequente rompimento (6). Foi também demonstrado que a angiotensina II (ANG II, o principal efetor do SRA), via receptores AT1, tem papel importante nesta patologia, modulando diretamente a função das células endoteliais da BHE e alterando a sua permeabilidade $(9,19,20)$

Trabalhos recentes mostraram que a infusão subcutânea prolongada de ANG II, mesmo em doses subpressoras, causa inflamação dos vasos cerebrais, levando à adesão leucocitária e estresse oxidativo, os quais encontravam-se associados a aumento na permeabilidade da BHE $(13,21)$. Foi também demonstrado que a inibição dos receptores AT1 bloqueia o aumento da permeabilidade celular e reduz o edema cerebral relacionados com a hipertensão arterial, mesmo na ausência da redução dos níveis pressóricos, sugerindo um papel crucial do sistema renina-angiotensina (SRA), e em especial da ANG II, em modular o funcionamento da BHE $(19,22,23)$. Sabe-se que o SRA desempenha importante papel na manutenção da pressão arterial (PA) e na homeostase de fluidos corporais através de ações tanto a nível periférico quanto central (9). Por outro lado, muitos trabalhos da literatura têm demonstrado que a hiperatividade do SRA encefálico, característica da hipertensão arterial, é um importante fator casual de disfunção autonômica e hipertonia simpática, acompanhadas por importantes déficits no controle cardiovascular (24-26). Em conjunto essas observações nos levam a supor que um dos mecanismos pelos quais a ANG II medeia o desequilíbrio autonômico seria através da lesão da BHE.

Uma série de trabalhos de nosso e outros laboratórios têm demonstrado que o treinamento aeróbio de baixa a moderada intensidade é uma importante conduta terapêutica para reduzir significativamente a atividade do SRA encefálico, reverter a 
disfunção autonômica e corrigir déficits funcionais da circulação na hipertensão e mesmo em indivíduos portadores de insuficiência cardíaca (27-31). Estas evidências experimentais nos levaram a propor que um dos mecanismos através do qual o treinamento aeróbio facilitaria o controle autonômico da circulação seria a melhora substancial da circulação encefálica, mediada pela correção da lesão da BHE frente à redução do SRA nos hipertensos treinados.

Trabalho recente de nosso laboratório investigando a microcirculação em áreas autonômicas do SNC têm indicado que o treinamento aeróbio (T) de SHR na fase mantida da hipertensão reduz o extravasamento da BHE nos núcleos paraventricular do hipotálamo (PVN, Figura 1, (32)), do trato solitário (NTS) e rostroventrolateral do bulbo (RVLM).

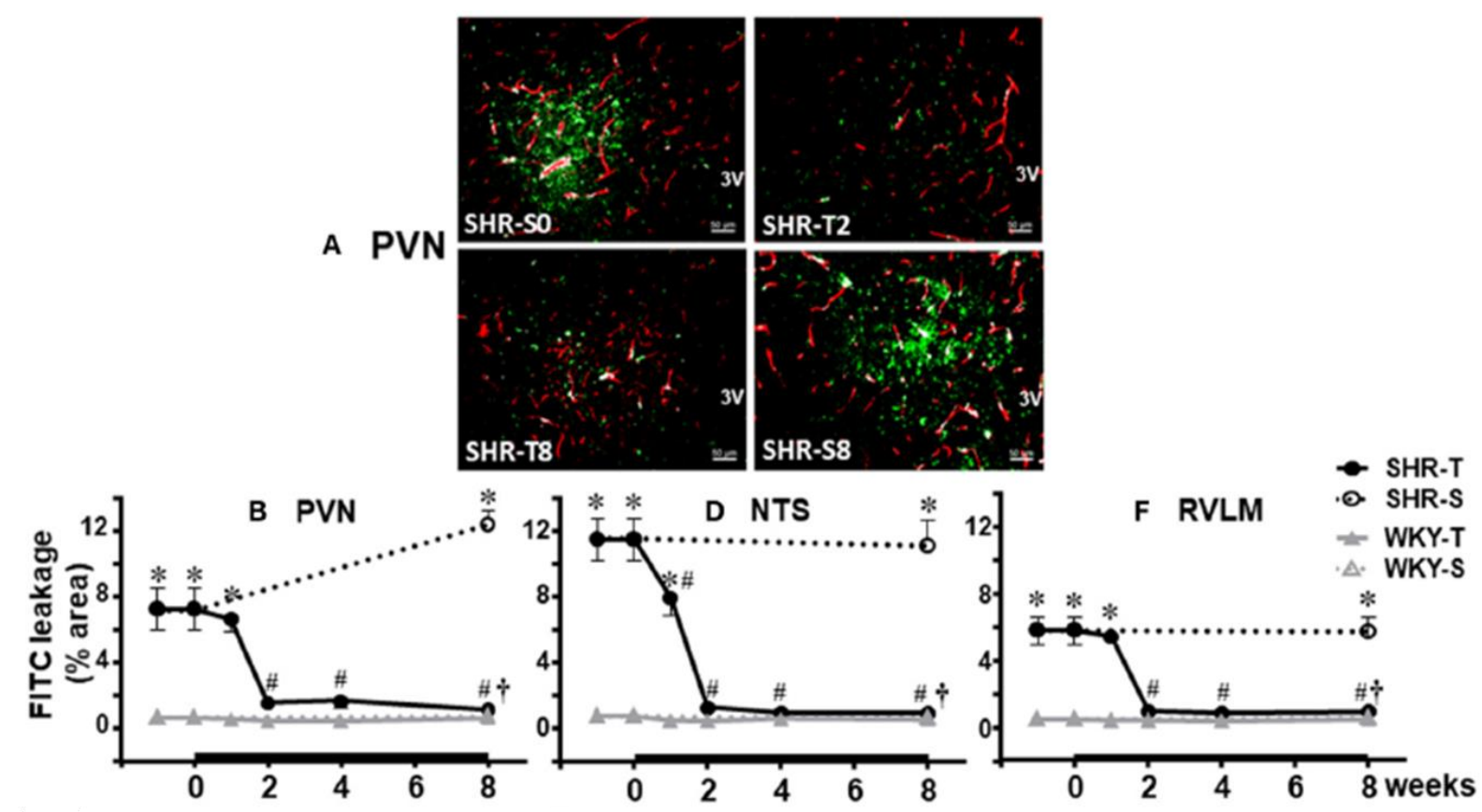

Figura 1. Evolução temporal da porcentagem de extravasamento do FITC-dextran (10kD) no PVN, NTS e RVLM dos grupos SHR e WKY submetidos a 8 semanas de treinamento (T) ou sedentarismo (S). Significâncias $(\mathrm{P}<0,05)$ : * vs. WKY $\dagger$ vs. sedentário \# vs. semana 0 (reproduzido de (32).

Nos SHR treinados a pronta redução do extravasamento do FITC-dextran de baixo peso molecular injetado intra-arterial (2a semana experimental) coincide temporalmente com a redução da expressão de angiotensinogênio no PVN, NTS e RVLM (CHAAR et al, 2015), com a normalização do controle reflexo da circulação e com a redução da razão LF/HF para o coração, também observadas na $2^{\mathrm{a}}$. semana experimental (MASSON et al, 2014). Estes achados experimentais reforçam nossa proposição anterior de que a correção da integridade da BHE possa ser um dos mecanismos determinantes 
dos efeitos benéficos do treinamento aeróbio sobre o controle autonômico da circulação em hipertensos treinados. Experimentos em andamento no laboratório têm ainda indicado que a infusão intracerebroventricular de ANG II nos SHR simultaneamente ao treinamento aeróbio reduz significantemente a melhora observada na função da BHE após treinamento.

Os dados acima apresentados comprovam a lesão da BHE em SHR na fase crônica da hipertensão, sua correção pelo treinamento aeróbio e o envolvimento do SRA (e em especial da ANG II) em ambas as situações. Não indicam, no entanto, quais dos constituintes de BHE estariam comprometidos na lesão observada nos SHR sedentários, assim como em que estruturas o treinamento age para reduzir/corrigir o extravasamento da BHE. Pretendemos, em continuidade à nossa linha de pesquisa, investigar em SHR sedentários e treinados e seus controles normotensos pareados por idade (WKY), a expressão dos diferentes constituintes da BHE. São, portanto, objetivos deste trabalho investigar no PVN, importante área encefálica de controle autonômico, de SHR e WKY, treinados ou mantidos sedentários, a expressão gênica/proteica dos diferentes componentes da BHE (e os mecanismos de transporte por eles condicionados) envolvidos na mediação das respostas cardiovasculares à hipertensão e ao treinamento aeróbio.

\section{CONCLUSÕES}

Nossos resultados mostraram que a disfunção autonômica que caracteriza a fase crônica da hipertensão ocorre simultaneamente ao aumento da permeabilidade da BHE determinada pelo aumento da expressão de vesículas transendoteliais positivas para a caveolina-1 que condicionam a transcitose, sem alterações dos constituintes da barreira e do transporte paracelular.

O treinamento aeróbio de baixa a moderada intensidade diminui e normaliza a permeabilidade da BHE de hipertensos por reduzir a transcitose e a expressão de vesículas transendoteliais, aumentando adicionalmente a função de barreira por reforçar as junções oclusivas pela maior expressão de claudina-5 e por aumentar a densidade dos podócitos dos astrócitos que revestem os capilares do PVN ventromedial. Estas adaptações induzidas pelo treinamento nesta importante área de integração cardiovascular melhoram a perfusão tecidual e contribuem substancialmente para normalizar a disfunção autonômica mesmo na persistência da hipertensão. 



\section{Referências bibliográficas}

1. Tolkien JR. The Council of Elrond. In: The Lord of the Rings: The Fellowship of the Ring. 1954.

2. Bradbury MW. The Blood-brain barrier. Exp Physiol. 1993;78:453-72.

3. Abbott NJ, Rönnbäck L, Hansson E. Astrocyte-endothelial interactions at the blood-brain barrier. Nat Rev Neurosci. 2006;7(1):41-53.

4. Daneman R, Prat A. The Blood - Brain Barrier. Cold Spring Harb Perspect Biol. 2015;7:1-5.

5. Armulik A, Genové G, Mäe M, Nisancioglu MH, Wallgard E, Niaudet C, et al. Pericytes regulate the blood-brain barrier. Nature. 2010;468(7323):557-61.

6. Lawther BK, Kumar S, Krovvidi H. Blood-brain barrier. Contin Educ Anaesthesia, Crit Care Pain. 2011;11(4):128-32.

7. Tornavaca O, Chia M, Dufton N, Almagro LO, Conway DE, Randi AM, et al. ZO-1 controls endothelial adherens junctions, cell-cell tension, angiogenesis, and barrier formation. J Cell Biol. 2015;208(6):821-38.

8. DE Vries HE, Kuiper J, Boer AGDE, Berkel TJCVAN, Breimer DD. The BloodBrain Barrier in Neuroinflammatory Diseases. Pharmacol Rev. 1997;49(2).

9. Fleegal-DeMotta MA, Doghu S, Banks WA. Angiotensin II modulates BBB permeability via activation of the AT(1) receptor in brain endothelial cells. $J$ Cereb Blood Flow Metab. 2009;29(3):640-7.

10. Wilhelm I, Krizbai IA. In Vitro Models of the Blood-Brain Barrier for the Study of Drug Delivery to the Brain. Mol Pharm. 2014 Jul 7;11(7):1949-63.

11. Miller DS. Regulation of P-glycoprotein and other ABC drug transporters at the blood-brain barrier. Trends Pharmacol Sci. 2010;31(6):246-54.

12. Faraco G, Iadecola C. Hypertension: A harbinger of stroke and dementia. Hypertension. 2013;62(5):810-7.

13. Zhang M, Mao Y, Ramirez SH, Tuma RF, Chabrashvili T. Angiotensin II induced cerebral microvascular inflammation and increased blood-brain barrier permeability via oxidative stress. Neuroscience. 2010;171(3):852-8.

14. Ueno M, Chiba Y, Matsumoto K, Murakami R, Fujihara R, Kawauchi M, et al. Blood-brain barrier damage in vascular dementia. Neuropathology. 2015;(August):n/a-n/a.

15. Hill J, Rom S, Ramirez SH, Persidsky Y. Emerging Roles of Pericytes in the 
Regulation of the Neurovascular Unit in Health and Disease. J Neuroimmune Pharmacol. 2014;9(5):591-605.

16. Zlokovic B V. The Blood-Brain Barrier in Health and Chronic Neurodegenerative Disorders. Neuron. 2008;57(2):178-201.

17. Hom S, Fleegal MA, Egleton RD, Campos CR, Hawkins BT, Davis TP. Comparative changes in the blood-brain barrier and cerebral infarction of SHR and WKY rats. Am J Physiol Regul Integr Comp Physiol. 2007;292(5):R188192.

18. Mueller SM, Heistad DD. Effect of chronic hypertension on the blood-brain barrier. Hypertension. 1980;2(6):809-12.

19. Biancardi V, Stern J. Compromised blood-brain barrier permeability: Novel mechanism by which circulating angiotensin II signals sympathoexcitatory centers during hypertension. J Physiol. 2015;53:n/a-n/a.

20. Pelisch N, Hosomi N, Mori H, Masaki T, Nishiyama A. RAS inhibition attenuates cognitive impairment by reducing blood- brain barrier permeability in hypertensive subjects. Curr Hypertens Rev. 2013;9(2):93-8.

21. Capone C, Faraco G, Park L, Cao X, Davisson RL, Iadecola C. The cerebrovascular dysfunction induced by slow pressor doses of angiotensin II precedes the development of hypertension. Am J Physiol Heart Circ Physiol. 2011;300(1):H397-407.

22. Ito H, Takemori K, Kawai J, Suzuki T. AT1 Receptor Antagonist Prevents Brain Edema Without Lowering Blood Pressure. In: Mendelow AD, Baethmann A, Czernicki Z, Hoff J, Ito U, James H, et al., editors. Brain Edema XI SE - 29. Springer Vienna; 2000. p. 141-5. (Acta Neurochirurgica Supplements; vol. 76).

23. Biancardi V, Son SJ, Ahmadi S, Filosa J a, Stern JE. Circulating angiotensin II gains access to the hypothalamus and brain stem during hypertension via breakdown of the blood-brain barrier. Hypertension. 2014;63(3):572-9.

24. de Kloet AD, Liu M, Rodríguez V, Krause EG, Sumners C. Role of neurons and glia in the CNS actions of the renin-angiotensin system in cardiovascular control. Am J Physiol - Regul Integr Comp Physiol. 2015 Sep 1;309(5):R444-58.

25. Veerasingham SJ, Raizada MK. Brain renin-angiotensin system dysfunction in hypertension: recent advances and perspectives. Br J Pharmacol. 2003;139(2):191-202.

26. Diz DI, Arnold AC, Nautiyal M, Isa K, Shaltout H a, Tallant EA. Angiotensin 
peptides and central autonomic regulation. Curr Opin Pharmacol. 2011;11(2):131-7.

27. Chaar LJ, Alves TP, Batista Junior AM, Michelini LC. Early Training-Induced Reduction of Angiotensinogen in Autonomic Areas-The Main Effect of Exercise on Brain Renin-Angiotensin System in Hypertensive Rats. PLoSONE. 2015;10(1932-6203 (Electronic)):e0137395.

28. Felix JVC, Michelini LC. Training-induced pressure fall in spontaneously hypertensive rats is associated with reduced angiotensinogen mRNA expression within the nucleus tractus solitarii. Hypertension. 2007;50(4):780-5.

29. Masson GS, Costa TSR, Yshii L, Fernandes DC, Soares PPS, Laurindo FR, et al. Time-dependent effects of training on cardiovascular control in spontaneously hypertensive rats: role for brain oxidative stress and inflammation and baroreflex sensitivity. PLoS One. 2014 Jan;9(5):e94927.

30. Agarwal D, Welsch M, Keller J, Francis J. Chronic exercise modulates RAS components and improves balance between pro- and anti-inflammatory cytokines in the brain of SHR. Basic Res Cardiol. 2011;106(6):1069-85.

31. Zheng H, Sharma NM, Liu X, Patel KP. Exercise training normalizes enhanced sympathetic activation from the paraventricular nucleus in chronic heart failure: role of angiotensin II. Am J Physiol Regul Integr Comp Physiol. 2012;303(4):R387-94.

32. Buttler L, Jordão MT, Fragas MG, Ruggeri A, Ceroni A, Michelini LC. Maintenance of blood-brain barrier integrity in hypertension: A novel benefit of exercise training for autonomic control. Front Physiol. 2017;8:1-14.

33. Cavalleri MT, Burgi K, Cruz JC, Jordao MT, Ceroni a., Michelini LC. Afferent signaling drives oxytocinergic preautonomic neurons and mediates traininginduced plasticity. AJP Regul Integr Comp Physiol. 2011;301(4):R958-66.

34. Coimbra R, Sanchez LS, Potenza JM, Rossoni L V., Amaral SL, Michelini LC. Is gender crucial for cardiovascular adjustments induced by exercise training in female spontaneously hypertensive rats? Hypertension. 2008;52(3):514-21.

35. Silva Jr SD, Zampieri TT, Ruggeri A, Ceroni A, Aragão DS, Fernandes FB, et al. Downregulation of the vascular renin-angiotensin system by aerobic training: focus on the balance between vasoconstrictor and vasodilator axes. Circ J. 2015;79(6):1372-80.

36. Tietz S, Engelhardt B. Brain barriers: Crosstalk between complex tight junctions 
and adherens junctions. J Cell Biol. 2015;209(4):493-506.

37. Okamoto T, Schlegel a, Scherer PE, Lisanti MP. Caveolins, a family of scaffolding proteins for organizing \&quot;preassembled signaling complexes\&quot; at the plasma membrane. J Biol Chem. 1998;273(10):5419-22.

38. Lisanti MP, Scherer PE, Tang Z, Sargiacomo M. Membrane domains. Trends Cell Biol. 1994;4:231-235.

39. Andreone BJ, Chow BW, Tata A, Lacoste B, Ben-Zvi A, Bullock K, et al. BloodBrain Barrier Permeability Is Regulated by Lipid Transport-Dependent Suppression of Caveolae-Mediated Transcytosis. Neuron. 2017;94:581-94.

40. Chen ZL, Yao Y, Norris EH, Kruyer A, Jno-Charles O, Akhmerov A, et al. Ablation of astrocytic laminin impairs vascular smooth muscle cell function and leads to hemorrhagic stroke. J Cell Biol. 2013;202(2):381-95.

41. Yao Y, Chen Z-L, Norris EH, Strickland S. Astrocytic laminin regulates pericyte differentiation and maintains blood brain barrier integrity. Nat Commun. $2014 ; 5: 3413$.

42. Stratman AN, Malotte KM, Mahan RD, Davis MJ, Davis GE. Pericyte recruitment during vasculogenic tube assembly stimulates endothelial basement membrane matrix formation. Blood. 2009;114(24):5091-101.

43. Joutel A, Faraci FM. Cerebral Small Vessel Disease: Insights and Opportunities From Mouse Models of Collagen IV-Related Small Vessel Disease and Cerebral Autosomal Dominant Arteriopathy With Subcortical Infarcts and Leukoencephalopathy. Stroke. 2014;45:1215-21.

44. Nedergaard M, Ransom B, Goldman S a. New roles for astrocytes: Redefining the functional architecture of the brain. Trends Neurosci. 2003;26(10):523-30.

45. Rash JE, Yasumura T, Hudson CS, Agre P, Nielsen S. Direct immunogold labeling of aquaporin-4 in square arrays of astrocyte and ependymocyte plasma membranes in rat brain and spinal cord. Proc Natl Acad Sci U S A. 1998;95(20):11981-6.

46. Abbott NJ, Rönnbäck L, Hansson E. Astrocyte-endothelial interactions at the blood-brain barrier. Nat Rev Neurosci. 2006;7(1):41-53.

47. Keaney J, Campbell M. The dynamic blood-brain barrier. FEBS J. 2015;282(21):4067-79.

48. Hagan N, Ben-Zvi A. The molecular, cellular, and morphological components of blood-brain barrier development during embryogenesis. Semin Cell Dev Biol. 
$2015 ; 38: 7-15$.

49. Al-Sarraf H, Ghaaedi F, Redzic Z. Time course of hyperosmolar opening of the blood-brain and blood-CSF barriers in spontaneously hypertensive rats. J Vasc Res. 2007;44(2):99-109.

50. Krueger M, Härtig W, Reichenbach A, Bechmann I, Michalski D. Blood-brain barrier breakdown after embolic stroke in rats occurs without ultrastructural evidence for disrupting tight junctions. PLoS One. 2013;8(2):e56419.

51. Iadecola C. The Pathobiology of Vascular Dementia. Neuron. 2013;80(4):84466.

52. Ueno M, Sakamoto H, Tomimoto H, Akiguchi I, Onodera M, Huang CL, et al. Blood-brain barrier is impaired in the hippocampus of young adult spontaneously hypertensive rats. Acta Neuropathol. 2004;107(6):532-8.

53. Mohammadi MT, Dehghani GA. Acute hypertension induces brain injury and blood-brain barrier disruption through reduction of claudins mRNA expression in rat. Pathol Res Pract. 2014;210(12):985-90.

54. Pelisch N, Hosomi N, Ueno M, Nakano D, Hitomi H, Mogi M, et al. Blockade of AT1 Receptors Protects the Blood-Brain Barrier and Improves Cognition in Dahl Salt-Sensitive Hypertensive Rats. Am J Hypertens. 2011;24(3):362-8.

55. Nitta T, Hata M, Gotoh S, Seo Y, Sasaki H, Hashimoto N, et al. Size-selective loosening of the blood-brain barrier in claudin-5-deficient mice. J Cell Biol. 2003;161(3):653-60.

56. Sofroniew M V. Reactive astrocytes in neural repair and protection. Neuroscientist. 2005;11(5):400-7.

57. Waki H, Gouraud SS, Maeda M, Raizada MK, Paton JFR. Contributions of vascular inflammation in the brainstem for neurogenic hypertension. Respir Physiol Neurobiol. 2011;178(3):422-8.

58. Barres BA. The Mystery and Magic of Glia: A Perspective on Their Roles in Health and Disease. Neuron. 2008;60(3):430-40.

59. Haseloff RF, Blasig IE, Bauer HC, Bauer H. In search of the astrocytic factor(s) modulating blood-brain barrier functions in brain capillary endothelial cells in vitro. Cell Mol Neurobiol. 2005;25(1):25-39.

60. Ceroni A, Chaar LJ, Bombein RL, Michelini LC. Chronic absence of baroreceptor inputs prevents training-induced cardiovascular adjustments in normotensive and spontaneously hypertensive rats. Exp Physiol. 2009;94(6):630- 
40.

61. Michelini LC, Stern JE. Exercise-induced neuronal plasticity in central autonomic networks: Role in cardiovascular control. Exp Physiol. 2009;94(9):947-60. 